\title{
Chemoprevention in Head \& Neck Cancer-A Mirage or Miracle
}

\section{Uma Garg}

Professor \& Head Department of ENT, B.P.S Govt. Medical College for Women, Khanpur Kalan, Sonipat Haryana Punjab, India

\section{Corresponding Author: Uma Garg}

Received: September 24, 2015; Accepted: October 01, 2015; Published: October 05, 2015

Keywords: Dura; Egr-1; Amyloid precursor protein; Transcriptional regulation; Alzheimer's disease

\section{Editorial}

Head \& neck cancers constitute the 6th most common cancer in the world more so in Asian subcontinent. Cancer is a life style disease rather than a genetic one. Only $5-10 \%$ of human cancers have genetic factors. Despite tremendous therapeutic advancements in the management of head \& neck cancers in the last decade, treatment with the curative intent is not able to improve the survival patterns. A thorough understanding of the biological history of cancer at the cellular level raises a pertinent question in the minds of all concerned, whether cancer could be prevented at the level of cell growth \& division. Sincere efforts are on to achieve the same. The term Chemoprevention was coined by Sporn in 1976 \& has been considered a recent \& novel tool to fight this dreadful disease. Chemoprevention is still under investigations and appears to be an appealing strategy. Chemo preventive agents are naturally occurring, biological or synthetic chemicals which can be utilized to arrest or suppress or to prevent the progression of carcinogenesis. These compounds may be dietary or in the form of supplements or these belong to diverse chemical classes \& when used as potential chemo preventive agents not only help in prevention of development of the cancer but also help the patients with cancer in controlling the emergence of a second tumor or reccurence. Primary chemoprevention is directed at the subjects with premalignant lesions namely keratosis with atypia, leukoplakia or erythroplakia. Secondary chemoprevention is targeted at patients with cancer in order to prevent recurrence and second primary while they are undergoing potentially curative therapy. Primary chemoprevention can be extended to involve the group at risk but otherwise healthy individual's e.g. chronic smokers. Secondary prevention is also known as adjuvant prevention. Different randomized clinical trials \& studies on association between intake of foods rich in such agents $\&$ cancer have yielded mixed results. Therefore it is imperative to understand the mechanism of cancer development at the cell division level \& to arrest it there. The ability of these dietary agents to sensitize the cancer cells to different chemotherapeutic agents \& radiotherapy $\risingdotseq$ drumamkgarg_1962@yahoo.co.in

Professor \& Head Department of ENT, B.P.S Govt. Medical College for Women, Khanpur Kalan, Sonipat Haryana Punjab

\section{Tel: +919216444268}

Citation: Garg U.Chemoprevention in Head \& Neck Cancer-A Mirage or Miracle. Head Neck Cancer Res. 2015, 1:1.

thus improving the results has been demonstrated in carcinoma breast $\&$ cervix in various research articles (K. Sahin et al). These studies suggest inhibition of breast cancer cell proliferation at G2/M stage of cell cycle in a dose dependent manner \& also augment the effects of radiotherapy. Similarly experiments on rats have shown preventive effects of magnesium hydroxide on carcinogenesis on giving magnesium hydroxide alone \& along with a carcinogenic agent in diet. The first group had lower incidence of large bowel neoplasm than the second supporting the protective effect of magnesium as a chemo preventive agent. Soy isoflavone, a soy derivative sensitizes the cervical cancer cells to Cisplatin promoting recovery \& also mitigating side effects. These substances have been believed to reverse chemo resistance $\&$ radioresistance in patients undergoing therapy. These chemo preventive agents can be given safely as complementary therapy in advanced cancers as an adjunct to standard cancer therapy without causing adverse effects on normal tissues. The aim of this presentation is to sensitize all concerned with care of a cancer patient regarding Chemoprevention as a promising tool to control cancer particularly in head \& neck region.

\section{Cell Cycle (Stages)}

Cancer is a multistep, multiyear, complex process affected by a multitude of factors acting concurrently in the etiopathogenesis of the disease. These multistep somatic/phenotypic and genotypic changes keep on accumulating in the cells and may not be sequentially additive but may be chaotic and arise from diversified pathways. Also direct, predictable and consistent 
correlation between clinical and histological features may be absent. So, human cancer is a long process where conversion of normal mucosa to carcinoma via hyperplasia and dysplasia may take as long as 30 years. Cell cycle at all its stages is regulated strictly by a group of specialised proteins that interact with each other \& external stimuli during specific sequence of events leading to cell division, proliferation or death. These stages are G1, S, G2 \& M. After M or mitosis the new cell enters interphase or resting phase. A young cell enters the reproductive phase in G1 phase where all the cellular components in the cell excluding the chromosomes are duplicated. Then in next phase i.e. $S$ phase each of the 23 pairs of chromosomes duplicate. Now this process of duplication can be affected by external agents like viruses, UV light \& tobacco. In the next phase i.e. G2 there are checks \& balances of the duplicated chromatin material or genetic formula for any errors if occurred during the preceding phase. Followed by deficiency/excess of certain materials will result in no repair of errors \& lead to formation of a defective daughter cell in next i.e. $M$ phase. Further proliferation if occurs unabated or uncontrolled will lead to cancerous development. If in $\mathrm{G} 2$ phase some useful things are added in the form of chemopreventive agents, possibly repair may happen $\&$ new cell is healthy $\&$ there is no disordered growth. Now the two daughter cells either goes to G0 phase/ resting phase or G1 phase. One in G0 carries out the function of that particular cell, functions till it dies. Resting phase means it will never enter the cell cycle again and becomes a stem cell waiting for a signal to enter cell cycle again to grow \& divide. At all these stages, process can be modified by certain factors which hamper or augment the process leading into a normal or abnormal growth i.e. cancer. Various mechanisms proposed in favor of their beneficial effects are suppression of cancer cell proliferation, inhibition of growth factor signaling pathway, induction of apoptosis, inhibition of angiogenesis, inhibition of cyclooxygenase-2 \& expression of antiapoptotic pathways. So the big Question is whether it is possible to identify these factors \& to exploit them for preventing or treating cancer. Research is continuing for the same. These proteins/factors if favorable are called tumor growth factors or oncogenes or if suppress the growth known as tumor suppressor proteins. Few of these have been identified $\&$ used to grow cells in vitro. The good proteins lead to an ordered or regulated growth with limited divisions \& the result is normal or may be a benign hypertrophy. The bad proteins result in a way \& uncontrolled division resulting into cancer. $\mathrm{S}$ phase is the stage of synthesis of genetic material. This DNA duplication/replication/proof reading is crucial for genetic information which is to be transferred to the daughter cell in the next stage. This stage is having extreme fidelity \& is liable to be influenced by array of factors which can be environmental, nutritional like dietary, drugs, sunlight, tobacco, alcohol or infections. The tumor enhancing or tumor suppressing proteins may be in the body itself or may be present in various foods or drugs or supplements \& these interact with other external agents or internal milieu to bring about changes in the chromosomes $\&$ the change may be favorable or against the growth of a normal cell. This also includes tumor markers which show varied expressions in different tumors. But in chemoprevention we have intermediate markers which are raised in premalignant conditions rather than malignant lesions. This also encompasses the concept of neutraceuticals playing a role in chemoprevention. Neutraceuticals are the substances which are not traditionally recognized nutrients but known to have positive physiological effects in human body \& benefit against chronic diseases and cancers.

1.Various agents identified in this regards are listed as follows:

\section{Lycopene}

3.Garcinol, Phenols

4.Isoflavones

5.Resveratrol

6.Selenium, magnesium

7.Gingerol

8.Curcumin

9.Cucurbitacin B

10.Folic acid

11.Capsaicin

12. Ascorbat

Since cancer is essentially a long process, may take years to develop it can be controlled at all its stages of progress. Experiments are on at various levels in laboratories to identify these substances $\&$ *put these into trial phases. Results with isolated compounds have been disappointing \& probably combinations prove better. Patient education seems to of paramount importance. The sequential steps of carcinogenesis can be arrested or inhibited by lifestyle modifications, dedications \& periodic regular screenings to stratify high risk groups which can then be followed up in cancer awareness programmes. As a part of health education Flag signs of malignancy should be displayed at entry, exit \& waiting areas of public places. Regular cancer clinics should be a part of all clinical establishments. Mandatory registration of cancer will prove instrumental in controlling \& preventing this maligning disease with A Dysregulated Life Style You Are Maligning the Internal Milieu Which Can Lead to Malignacy and It Can Be Regulated. 


\section{References}

1 Raffoul JJ, Kucuk O, Sarkar FH (2012) Dietary agents in cancer chemoprevention and treatment. Oncol J 749310.

2 Sahin K, Ibrahim HO, Academir F, Onderci M (2008) Chemoprevention of fibroid tumors by epigallocatechin-3-gallate in quail. Nutrition Research 28: 92-97.

3 Saadat N, Oncol J (2012) Potential role of Garcinol as anti-cancer agent 647206

4 Chow AY (2010) Cell cycle control by Oncogens and Tumor Suppressers: Driving the Transformation of Normal Cells into cancerous cells. Nature Education 3: 7.
5 Mori H, Morishita Y, Shinoda T, Tanaka T (1993) Preventive effect of magnesium hydroxide on carcinogen induced large bowel carcinogenesis in rats. Basic Life Sci 61: 111-8.

6 Dorai T, Aggarwal B (2004) Role of chemopreventive agents in cancer therapy. Cancer Lett. 215: 129-40.

7 Dong Feng Tan (2001) MCM2-A promising marker of pre-malignant regions of the lung a cohort study. BMC Cancer. 1: 6.

8 Ning Li, Zheng Sun, Chi Han, Zunshi Chan (1999) The chemopreventing effects of Tea on Human Oral Pre-Cancerous mucosal lesions. Experimental Biology and Medicine. 220: 218-224.

9 Tsao AS, Kim ES, Hong WK, Clin J (2004) Chemoprevention of Cancer. CA Cancer 54: 150-180. 